Quim. Nova, Vol. 31, No. 5, 1190-1198, 2008

\title{
VALIDAÇÃo DE MÉTODOS CROMATOGRÁFICOS PARA A DETERMINAÇÃO DE RESÍDUOS DE MEDI- CAMENTOS VETERINÁRIOS EM ALIMENTOS
}

\author{
Jonas Augusto Rizzato Paschoal e Susanne Rath* \\ Departamento de Química Analítica, Instituto de Química, Universidade Estadual de Campinas, CP 6154, 13084-971 Campinas \\ - SP, Brasil \\ Flavia Pereira da Silva Airoldi e Felix G. R. Reyes \\ Departamento de Ciência de Alimentos, Faculdade de Engenharia de Alimentos, Universidade Estadual de Campinas, CP 6121, \\ 13084-971 Campinas - SP, Brasil
}

Recebido em 2/4/07; aceito em 20/9/07; publicado na web em 9/4/08

\begin{abstract}
VALIDATION OF CHROMATOGRAPHIC METHODS FOR THE DETERMINATION OF RESIDUES OF VETERINARY DRUGS
IN FOODS. Different agencies that supply validation guidelines worldwide establish almost the same parameters to be evaluated in the validation process of bioanalytical methods. However, they recommend different procedures, as well as establish different acceptance criteria. The present review delineates and discusses the stages involved in the validation procedures of bioanalytical methods designed for determining veterinary residues in food, explaining the main differences in the guidelines established for this purpose by the main regulatory agencies in the world.
\end{abstract}

Keywords: validation; veterinary residues in food; bioanalytical methods.

\section{INTRODUÇÃO}

O cenário atual mundial da produção de alimentos revela o grande interesse da sociedade quanto à inocuidade do produto final a ser consumido. Afamados acontecimentos envolvendo a contaminação de alimentos, como foi o caso de benzeno na água e sucos de frutas, nitrofuranos em carne de frango, cloranfenicol no mel, entre outros, há muito têm despertado a atenção do consumidor. ${ }^{1}$ Este quadro tem forçado os países a estabelecerem normas legislativas rígidas que garantam o consumo de alimentos seguros por parte da população.

Métodos analíticos têm sido desenvolvidos para a determinação de resíduos de contaminantes em alimentos como ferramenta principal para assegurar que os produtos estejam enquadrados nas determinações legais. Para que estes métodos garantam a disponibilidade de um alimento seguro, é preciso que sejam normalizadas e cumpram requisitos que garantam bons resultados. Com o objetivo de assegurar a confiabilidade dos resultados obtidos, são delineados procedimentos de validação do processo analítico empregado, como garantia da qualidade das medições químicas, através da sua comparabilidade, rastreabilidade e confiabilidade.

Os laboratórios que realizam as análises em alimentos precisam ter capacidade técnica comprovada para tal compromisso, a qual é garantida pelas normas de credenciamento estabelecidas por órgãos responsáveis, que no Brasil são representados pela Agência Nacional de Vigilância Sanitária (ANVISA) ${ }^{2}$ e pelo Instituto Nacional de Metrologia, Normalização e Qualidade Industrial (INMETRO), ${ }^{3}$ que disponibilizam guias para procedimentos de validação de métodos analíticos (Resolução ANVISA RE nº 899, de 29/05/2003, ${ }^{4}$ e o documento INMETRO DOQ-CGCRE-008, de 03/2003, ${ }^{5}$ respectivamente). Há ainda órgãos internacionais, como União Internacional de Química Pura e Aplicada (IUPAC), ${ }^{6}$ Organização Internacional para Padronização (ISO), ${ }^{7}$ Conferência Internacional em Harmonização (ICH), ${ }^{8}$ que também estabelecem

*e-mail: raths@iqm.unicamp.br procedimentos de validação como critério fundamental no credenciamento de laboratórios.

Há tempo o assunto de medidas de incerteza em química analítica tem gerado significativo interesse e discussão. Sabe-se que o resultado analítico não pode ser avaliado sem uma estimativa de medidas de incerteza para comparar com a convicção requerida. O Guia de Expressão de Incertezas em Medidas (GUM) publicado pela ISO ${ }^{9}$ estabelece regras para avaliação e expressão dessas incertezas para uma grande variedade de medidas. Esse guia foi interpretado no contexto da química analítica pela EURACHEM ${ }^{10}$ - grupo de organizações européias que buscam convergir as tendências internacionais em química analítica para esse continente - em 1995.

No que diz respeito às determinações de contaminantes em alimentos, as técnicas cromatográficas de separação destacam-se no âmbito analítico pela reconhecida capacidade de possibilitarem análises qualitativas e quantitativas. Diante disto, a presente revisão teve como objetivos delinear e discutir as etapas envolvidas no procedimento de validação de métodos bionanalíticos para a determinação de resíduos de medicamentos veterinários em alimentos, esclarecendo as principais diferenças dos principais guias elaborados pelas diferentes agências reguladoras oficiais, entre essas a ANVISA, a Agência dos Estados Unidos que regula sobre alimentos e drogas (FDA, Food and Drug Administration) e a Comunidade Européia (EC), e apontar e discutir as principais semelhanças e diferenças existentes entre elas.

De acordo com a ANVISA, os métodos cromatográficos destinados à determinação quantitativa de fármacos e/ou metabólitos em matrizes biológicas são inseridos no contexto dos métodos bioanalíticos. $^{4}$

\section{Contaminantes em alimentos e legislação}

O Codex Alimentarius é um Programa Conjunto da Organização das Nações Unidas para a Agricultura e a Alimentação (FAO) e da Organização Mundial da Saúde (OMS). Trata-se de um fórum internacional de normalização sobre alimentos, criado em 1963, 
com sede em Roma, e suas normas têm como finalidade proteger a saúde da população, assegurando práticas eqüitativas no comércio regional e internacional de alimentos, fomentando e coordenando todos os trabalhos que se realizam em normalização. Na década de 70, o Brasil tornou-se membro deste Programa e, em 1980, com a criação do Comitê do Codex Alimentarius do Brasil (CCAB), através das Resoluções 01/80 e 07/88 do CONMETRO (Conselho Nacional de Metrologia, Normalização e Qualidade Industrial), conseguiu uma articulação mais representativa do setor alimentício. O CCAB tem como principais finalidades a participação nos comitês internacionais do Codex Alimentarius e a defesa dos interesses nacionais, bem como a utilização das Normas Codex como referência para a elaboração e atualização da legislação e regulamentação nacional de alimentos. ${ }^{3}$

Quando se fala em resíduos de contaminantes em alimentos, fazse referência a uma grande variedade de substâncias. Há muito tempo a agricultura lança mão de artifícios envolvendo aplicação de produtos químicos durante o processo de produção, como os agroquímicos (agrotóxicos ou praguicidas), e mesmo a pecuária dispõe do uso de medicamentos veterinários, como antimicrobianos, promotores de crescimento e tranqüilizantes, entre outros. Essas substâncias podem remanescer no produto final na forma original ou, ainda, como subprodutos ou metabólitos, quando degradados ou biotransformados. O produto alimentício ainda pode conter toxinas produzidas por microrganismos eventualmente presentes (micotoxinas). A possibilidade da presença dessas substâncias no alimento representa um potencial risco à saúde do consumidor, de modo que métodos que garantam a isenção da presença de compostos tóxicos em alimentos se tornam cada vez mais imprescindíveis.

Métodos analíticos para resíduos de contaminantes tóxicos em alimentos requerem considerações técnicas especiais quando comparados a métodos delineados para outros fins, como em análises de medicamentos, por exemplo. Limites máximos de resíduos (LMR) específicos para cada analito alvo são estabelecidos, os quais devem ser levados em consideração no estabelecimento de um protocolo para validação do método analítico a ser empregado na determinação desses resíduos nos alimentos.

Para medicamento de uso veterinário, o LMR é definido como a concentração máxima de resíduo tolerável no alimento, resultante do seu uso. É baseado no tipo e quantidade de resíduo que não induz efeito adverso à saúde humana considerando-se a Ingestão Diária Aceitável (IDA) do composto. A IDA é a quantidade de uma substância que pode ser ingerida diariamente, durante toda a vida, sem que provoque danos à saúde. Ela é expressa em $\mathrm{mg} \mathrm{kg}^{-1}$ de peso corpóreo. A determinação da IDA é baseada nas informações toxicológicas disponíveis daquele composto na época da avaliação. ${ }^{11}$

Quando se estabelece um LMR, são levados em consideração os resíduos que ocorrem nos alimentos, assim como os provenientes do ambiente. No entanto, o LMR pode ser fundamentado em boas práticas no uso de medicamentos veterinários e nos métodos analíticos disponíveis.

No caso de substâncias proibidas, para as quais não há um LMR estabelecido, a Comunidade Européia (Diretiva 2002/657/EC) propõe o estabelecimento de um limite mínimo de performance requerida (LMPR), que representa a mínima quantidade da(s) substâncias(s) na amostra que pode ser detectada e confirmada. ${ }^{12}$

\section{VALIDAÇÃO DE MÉTODOS BIOANALÍTICOS}

\section{Requerimentos da pré-validação}

Em todos os tipos de métodos analíticos validados, há requerimentos de pré-validação que precisam estar em conformidade an- tes de se iniciar a validação do método. Alguns itens são de suma importância para assegurar uma boa validação e, consequientemente, a confiabilidade dos resultados. São eles: qualificação analítica do equipamento; conformidade do sistema e, estabilidade das soluções e amostras.

A qualificação analítica do equipamento envolve a adequação do equipamento frente aos resultados esperados. Para tanto, o equipamento deve estar em boa manutenção e, principalmente, calibrado. Para gerar resultados reprodutíveis e seguros as estabilidades das amostras, dos padrões, dos reagentes e das fases móveis precisam ser avaliadas antes do início da validação do método.

A seguir serão apresentadas considerações sobre procedimentos empregados para avaliar a conformidade do sistema e estabilidade das soluções e amostras.

\section{Conformidade do sistema}

Para o bom desenvolvimento de um procedimento de validação de um determinado método analítico é preciso assegurar que todo o sistema selecionado para o desenvolvimento do processo de análise esteja apto a fornecer resultados com precisão e exatidão aceitáveis e confiáveis. Esta é a etapa inicial a ser estabelecida, e é obtida para os métodos cromatográficos a partir de testes experimentais de conformidade do sistema (system suitability) que inclui avaliação dos seguintes parâmetros: fator de retenção $(k)$, fator de separação $(\alpha)$, resolução $(\mathrm{Rs})$, número de pratos $(\mathrm{N})$ e fator de assimetria (As).

A $\mathrm{ICH}^{13}$ inclui a etapa de avaliação da conformidade do sistema como parte integrante do processo de validação, ao passo que a Farmacopéia dos Estados Unidos (USP) ${ }^{14}$ a classifica como etapa preliminar ao procedimento de validação.

Um cromatograma característico com a discriminação das medidas que compõem os cálculos dos parâmetros cromatográficos é apresentado na Figura 1, onde $t_{M}$ indica o tempo que as moléculas do(s) analito(s) ficam na fase móvel; $t_{R}$ representa o tempo gasto desde a injeção do analito no sistema cromatográfico até a saída do ponto máximo do pico referente ao mesmo; $W_{b}$ é a largura da base do pico cromatográfico; $W_{50}$ é a largura do pico cromatográfico a meia altura.

A partir das medidas $t_{R}$ e $t_{M}$, pode-se obter o tempo de retenção relativo $\left(t^{\prime}{ }_{R}\right)$ que representa o tempo que o analito fica na fase estacionária (Equação 1):

$t^{\prime}=t_{R}-t_{M}$

A EC (Diretiva 2002/657/EC) ${ }^{12}$ recomenda que o $t_{R}{ }_{R}$ mínimo aceitável para um analito seja duas vezes $t_{M}$.

A seguir, são descritos os parâmetros cromatográficos avaliados na conformidade do sistema e suas respectivas equações: ${ }^{15}$

Fator de retenção $(k)$

É determinado pela razão dos tempos que as moléculas permanecem na fase estacionária $\left(t^{\prime}{ }_{R}\right)$ e na fase móvel $\left(t_{M}\right)$ (Equação 2).

$k=\frac{\left(t_{R}-t_{M}\right)}{t_{M}}=\frac{t_{R}^{\prime}}{t_{M}}$

Fator de separação $(\alpha)$

Dimensiona a separação relativa entre dois componentes consecutivos em um cromatograma, e é calculado pela razão entre os fatores de retenção dos dois picos adjacentes (1 e 2) (Equação 3):

$$
\alpha=\frac{\left(t_{R_{2}}-t_{M}\right)}{\left(t_{R_{1}}-t_{M}\right)}=\frac{t_{R_{2}}^{\prime}}{t_{R 1}^{\prime}}
$$




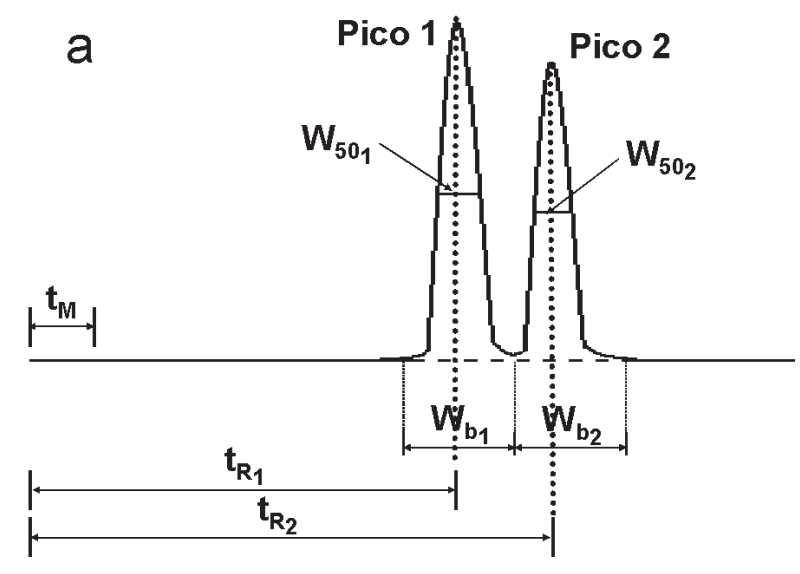

b

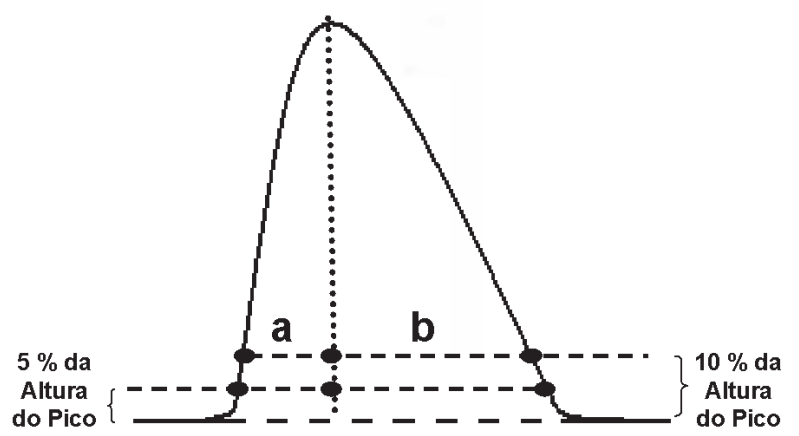

Figura 1. Medidas relacionadas à determinação dos parâmetros cromatográficos (adaptado da ref. 15): a) fatores de capacidade e de separação, resolução e número de pratos; b) fatores de assimetria e de alargamento

\section{Resolução (Rs)}

Assim como $\alpha, R s$ é um parâmetro de separação de dois componentes consecutivos em um cromatograma, calculada a partir da distância que separa os pontos máximos dos picos e da média das larguras de suas respectivas bases, ou das larguras na meia altura (Equação 4):

$$
R_{s}=2\left(\frac{t_{R_{2}}-t_{R_{1}}}{w_{b_{2}}+w_{b_{1}}}\right)=1,18\left(\frac{t_{R_{2}}-t_{R_{1}}}{w_{(50)_{2}}+w_{(50)_{1}}}\right)
$$

Quando $R s=1$, os dois picos são razoavelmente separados, com somente $2 \%$ de superposição se as quantidades dos dois componentes forem iguais. Maiores valores de resolução indicam melhor separação: $R s=1,25$ é suficiente para fins quantitativos, e $R s$ $>1,5$ indica separação completa. ${ }^{15}$

\section{Número de pratos $(N)$}

O cálculo de $N$ determina a eficiência de uma coluna, dimensionando a capacidade de interação da sua fase estacionária com cada um dos componentes de uma mistura que estejam eluindo através da mesma; é calculado para cada pico cromatográfico a partir do seu tempo de retenção e da largura do pico na linha de base $\left(W_{b}\right)$ ou à meia altura do pico $\left(W_{50}\right)$ (Equação 5):

$$
N=16 \frac{t_{R}^{\prime 2}}{w_{b}^{2}}=5,545\left(\frac{t_{R}^{\prime}}{w_{50}}\right)^{2}
$$

A eficiência pode ser afetada por vários fatores, incluindo as condições de análise, o tamanho da amostra, o tipo de soluto e, principalmente, o comprimento da coluna, fato que torna difícil uma comparação de $N$ entre colunas diferentes. Por isso, a avaliação comparativa entre colunas é feita pela medida da altura equivalente a um prato $(H)$, que corresponde à razão entre o comprimento da coluna, $L$, e $N$, eliminando a influência dos diferentes comprimentos da coluna (Equação 6) ${ }^{15}$ :

$$
H=\frac{L}{N}
$$

Fator de assimetria (As) elou de alargamento (TF)

Ambos medem a simetria e as distorções frontais ou posteriores (caudas) de um pico cromatográfico, sendo As calculado a $10 \%$ da altura do mesmo (Equação 7), e TF calculado a 5\% da altura (Equação 8):

$$
\begin{aligned}
A_{s} & =\frac{b}{a} \\
T_{F} & =\frac{a+b}{2 a}
\end{aligned}
$$

\section{Estabilidade}

A avaliação da estabilidade das soluções utilizadas no método analítico é de suma importância, já que uma degradação do analito ou dos constituintes da matriz durante a estocagem ou análise da amostra podem afetar a exatidão dos resultados.

Embora os objetivos sejam os mesmos, os protocolos recomendados para avaliar a estabilidade segundo a $\mathrm{EC}^{12}$ e FDA $^{16} / \mathrm{ANVISA}^{4}$ são diferentes. A seguir são apresentadas as recomendações adotadas por essas agências reguladoras.

A diretiva européia ${ }^{12}$ aponta para os seguintes procedimentos de avaliação da estabilidade do analito em solução e na matriz:

Estabilidade do analito em solução

Preparar a solução estoque do(s) analito(s) e diluir conforme especificado no método para uma concentração próxima ao limite máximo de resíduo (LMR) ou limite mínimo de performance requerida (LMPR). Dividir essa solução em quarenta alíquotas. Analisar uma alíquota imediatamente após o preparo $\left(C_{R}\right.$, concentração de referência). As demais alíquotas devem ser rotuladas e acondicionadas conforme esquema apresentado na Tabela 1.

Tabela 1. Esquema para determinação da estabilidade do(s) analito(s) em solução

\begin{tabular}{lccc}
\hline & $-20{ }^{\circ} \mathrm{C}$ & $+4{ }^{\circ} \mathrm{C}$ & $+20{ }^{\circ} \mathrm{C}$ \\
\hline Escuro & 10 alíquotas & 10 alíquotas & 10 alíquotas \\
Luz & & & 10 alíquotas \\
\hline
\end{tabular}

O tempo de estocagem pode ser selecionado como uma, duas, três e quatro semanas ou mais se necessário, como por exemplo, até quando o primeiro fenômeno de degradação for observado durante a identificação e/ou quantificação do(s) analito(s). O tempo máximo e as condições ótimas de estocagem devem ser registrados. O cálculo da concentração do(s) analito(s) remanescente(s) $\left(C_{r}\right)$ em cada uma das alíquotas depois de submetidas às diferentes condições testadas deve tomar como referência a concentração do analito obtida para aquela primeira alíquota analisada logo após o 
preparo das diluições $\left(C_{R}=100 \%\right)$ (Equação 9):

$$
C_{r}(\%)=\frac{C_{i} \times 100}{C_{R}}
$$

onde: $C_{i}$ é a concentração do analito sob a condição e no momento testados.

\section{Estabilidade do analito na matriz}

Empregar, quando possível, amostra que contenha o analito. No entanto, se a amostra não estiver disponível, deve ser empregada amostra branco fortificada com o analito.

Quando a amostra estiver disponível, a concentração do analito deve ser determinada na amostra ainda fresca. Além disso, alíquotas da amostra podem ser analisadas após 1, 2, 4 e 20 semanas, sendo que durante esse período as amostras devem ser estocadas a uma temperatura igual ou inferior a $-20{ }^{\circ} \mathrm{C}$.

Se não há a disponibilidade da amostra, utilizar uma amostra branco e homogeneizá-la. Dividir o material em 5 alíquotas. Fortificar cada alíquota com o analito, o qual deve estar preferencialmente preparado em uma pequena quantidade de solução aquosa. Analisar uma alíquota imediatamente. Estocar as alíquotas remanescentes a uma temperatura igual ou inferior a $-20{ }^{\circ} \mathrm{C}$ e analisálas após 1, 2, 4 e 20 semanas.

Para avaliar a estabilidade do(s) analito(s) na matriz, as agências FDA $^{16}$ e ANVISA ${ }^{4}$ estabelecem ensaios envolvendo ciclos de congelamento/descongelamento, em períodos de curta e longa duração, além de verificar também a estabilidade do(s) analito(s) em soluções padrão.

Para a estabilidade durante ciclos de congelamento e descongelamento a FDA recomenda congelar 3 alíquotas da amostra (amostra branco fortificada) em duas concentrações (baixa e alta) por $24 \mathrm{~h}$ à temperatura desejada e descongelar à temperatura ambiente; depois de descongeladas, as amostras devem ser novamente congeladas de 12 a $24 \mathrm{~h}$ sob as mesmas condições. Esse ciclo de congelamento/descongelamento deve ser repetido por mais duas vezes e, após o terceiro ciclo, as amostras devem ser analisadas. Se o analito não se apresentar estável, o mesmo procedimento deve ser repetido congelando as amostras a uma temperatura de $-70{ }^{\circ} \mathrm{C}$.

A estabilidade de curta duração é avaliada mediante análise de 3 alíquotas da amostra branco fortificada (concentração baixa e alta) depois de descongeladas e mantidas à temperatura ambiente por um período de 4 a $24 \mathrm{~h}$.

Para o ensaio da estabilidade de longa duração o tempo de armazenamento deve exceder o intervalo de tempo compreendido entre a coleta da primeira amostra e a análise da última.

A estabilidade das soluções padrão deve ser realizada à temperatura ambiente, após $6 \mathrm{~h}$ de sua preparação.

\section{Parâmetros analíticos da validação}

O procedimento de validação de métodos deve incluir todas as etapas necessárias para demonstrar que os resultados obtidos são confiáveis e reprodutíveis.

Uma questão de suma importância no delineamento das etapas a comporem o procedimento de validação de um método analítico está justamente no estabelecimento dessas etapas. A validação de um método desenvolvido, ou mesmo, a validação de modificações de ajuste de métodos já publicados, mesmo os oficiais, envolve uma etapa crítica que é o julgamento do nível de validação necessário conforme o objetivo a que se destina o método analítico. As diferentes agências reguladoras sugerem diferentes classificações para os níveis de validação de um método, todas diferenciadas con- forme a proposta a que se destina o mesmo.

A IUPAC destaca que no que diz respeito à análise de alimentos, os requerimentos para os métodos analíticos envolvem um exame das características de performance a partir de ensaios interlaboratoriais (que envolvem diferentes laboratórios, também conhecido como ensaio de proficiência, estudo colaborativo ou triagem colaborativa). Um número mínimo de laboratórios são requeridos para participarem dos estudos inter-laboratorias, preenchendo os requisitos para comporem o que a IUPAC classifica como procedimento de validação completa do método analítico (full validation). No entanto, nem sempre é prático ou necessário promover a validação completa de um método analítico. Em tais circunstâncias a IUPAC considera apropriado o que chama de validação do método em um único laboratório (single-laboratory validation). Essas circunstâncias podem ocorrer quando se pretende garantir a viabilidade do método antes de se proceder à validação completa, e/ou como etapa preliminar de avaliação de um método desenvolvido e na publicação de artigos científicos. ${ }^{17}$

A FDA também faz uso da classificação de validação completa e aponta sua necessidade quando se está desenvolvendo e implementando um método bioanalítico pela primeira vez, ou quando a análise envolve uma nova substância, ou quando metabólitos são adicionados a uma análise já existente para quantificação. Além da validação completa, a FDA ainda define outros dois níveis de classificação para os procedimentos de validação. Refere-se à validação parcial quando se pretende introduzir modificações em um método bioanalitico já validado, podendo envolver desde uma simples avaliação da exatidão e precisão intra-ensaios, até etapas que se aproximam de uma validação completa. Há ainda a validação cruzada (cross-validation) que envolve uma comparação dos parâmetros de validação quando dois ou mais métodos analíticos são usados para gerar dados dentro de um mesmo estudo ou através de estudos diferentes. Um exemplo de aplicação da validação cruzada se dá quando um método bioanalítico original validado é usado como referência para comparação com o método revisado. Este procedimento de validação pode também ocorrer em ensaios inter-laboratoriais. A validação cruzada também deve ser considerada quando resultados são gerados a partir de diferentes técnicas analíticas (por exemplo, LC-MS-MS vs ELISA). ${ }^{16}$

A $\mathrm{FAO}^{18}$ classifica os diferentes níveis de validação em face de uma diferenciação de quatro diferentes tipos de métodos analíticos (conforme delineamento do Codex Commodity Committees): (Tipo I) Métodos de definição; (Tipo II) Métodos de Referência; (Tipo III) Métodos Alternativos Aprovados e, (Tipo IV) Métodos de Tentativa. A FAO aponta ainda que a maioria dos métodos listada para resíduos de medicamentos veterinários se qualificaria, conforme a descrição apresentada, como Tipo IV (Métodos de Tentativa), enquanto apenas aqueles que tenham sido submetidos a um estudo colaborativo atenderiam aos requisitos para métodos Tipo I, II ou III.

Segundo o INMETRO, ${ }^{5}$ convém que um procedimento de ensaio seja coerente, claro, correto e tão completo quanto necessário, dentro dos limites estabelecidos pelo seu campo de aplicação. Um formato padronizado assegura que nenhum ponto importante tenha sido esquecido, que as informações a serem incluídas no procedimento sejam fornecidas sempre na mesma ordem e que qualquer assunto desejado possa ser encontrado rapidamente. Na padronização do formato dos métodos validados, o INMETRO sugere que se utilize a ABNT ISO/IEC Diretiva - Parte 3, a Norma ISO 78/2, além das prescrições da NBR ISO/IEC 17025.

$\mathrm{A} \mathrm{EC}^{12}$ e a ANVISA ${ }^{4}$ não apresentam classificações para os tipos de validação de métodos analíticos, atendo-se em seus documentos às definições dos parâmetros que compõem o procedimento geral de validação. 
Os parâmetros analíticos de validação (ou figuras de mérito) mais usados para avaliar métodos de separação são: especificidade/ seletividade, linearidade, sensibilidade, intervalo (faixa linear), precisão, limite de detecção, limite de quantificação, exatidão e robustez. A seguir são apresentadas as definições, em especial da IUPAC, e os procedimentos recomendados pela EC, FDA e ANVISA para cada um dos parâmetros de validação delineados para métodos desenvolvidos em um laboratório (single-laboratory validation), visando a determinação de resíduos de medicamentos veterinários em matrizes biológicas.

\section{Seletividade}

A seletividade é a capacidade de um método quantificar com exatidão o analito na presença de interferentes existentes na amostra. ${ }^{17}$ Esses interferentes podem ser substâncias quimicamente relacionadas com o analito, entre elas isômeros, metabólitos, substâncias endógenas, produtos de degradação, impurezas e outros.

As diretrizes da Comunidade Européia, ${ }^{12}$ ANVISA $^{4}$ e INMETRO $^{5}$ usam o termo especificidade como sinônimo de seletividade, o que pode levar a interpretações equivocadas. Por definição, a especificidade refere-se ao método que produz resposta para uma única substância, enquanto o termo seletividade se refere ao método que produz respostas para várias substâncias com uma característica em comum, mas que, no entanto, é capaz de distinguir umas das outras. Considerando que os métodos cromatográficos geralmente respondem para várias substâncias, o termo seletividade é mais apropriado.

A seletividade é um parâmetro que deve ser demonstrado e que depende do método analítico empregado. Para os métodos cromatográficos a seletividade é avaliada no sentido de garantir que o pico de resposta do analito (avaliado no tempo de retenção característico) seja proveniente exclusivamente do mesmo e não de outros compostos (interferentes) presentes na amostra. Para tanto, é conveniente a utilização de testes de pureza de pico com auxílio de detector de arranjo de fotodiodos ou espectrometria de massas.

As recomendações da $\mathrm{EC}^{12}$ para avaliar a seletividade de um método analítico são: selecionar compostos quimicamente relacionados ao analito (metabólitos, derivados, etc.) ou outras substâncias prováveis de estarem presentes com o(s) composto(s) de interesse nas amostras; analisar um número apropriado $(n \geq 20)$ de amostras isentas do(s) composto(s) de interesse (amostras branco) e verificar por qualquer interferência (sinal, picos, íons traços) na região esperada de eluição do analito de interesse; fortificar, a um nível relevante, amostras isentas do(s) composto(s) de interesse com substâncias que possam vir a interferir na identificação e quantificação do analito.

Após as análises, deve-se verificar se a presença de interferentes pode levar a uma identificação falsa; a identificação do analito alvo é prejudicada pela presença de um ou mais interferentes ou, a quantificação é notadamente influenciada pela presença de um ou mais interferentes.

A FDA ${ }^{16}$ recomenda que a seletividade seja avaliada mediante análise de amostras branco obtidas, no mínimo, de seis fontes diferentes da mesma matriz. Cada amostra branco deverá ser testada para interferentes e a seletividade deve ser garantida no limite inferior de quantificação (LIQ) ou lower limit of quantitation (LLOQ). Por definição, LIQ ou LLOQ é o menor nível quantificável com precisão e exatidão aceitáveis (ANVISA), ${ }^{4}$ que é a mesma definição do termo limite de quantificação (LOQ, limit of quantitation) recomendado pela IUPAC. ${ }^{17} \mathrm{O}$ INMETRO ${ }^{5}$ também emprega o termo limite de quantificação (LQ). Uma vez que não existe uma harmonização quanto a sigla para expressar o limite de quantificação, estaremos adotando nesta revisão o termo LOQ. O parâmetro limite de quantificação será discutido com maiores detalhes mais adiante.

No caso do emprego da cromatografia líquida associada à espectrometria de massa em tandem (LC-MS-MS), a FDA recomenda que seja também avaliado o efeito matriz. A ANVISA segue as recomendações da FDA e acrescenta que a resposta de picos interferentes no tempo de retenção do analito deve ser inferior a $20 \%$ da resposta do LOQ.

\section{Curva analítica, linearidade e sensibilidade}

Para qualquer método quantitativo, existe uma faixa de concentração do analito na qual o método pode ser aplicado. Segundo a ANVISA, a curva de calibração representa a relação entre a resposta do instrumento e a concentração conhecida do analito. ${ }^{4}$ Uma vez que o termo calibração é mais pertinente para instrumentos de medição e padrões, sugerimos, e estaremos empregando o termo curva analítica para expressar a relação sinal (resposta) em função da concentração do analito, em vez de curva de calibração como empregado pela ANVISA ${ }^{4}$ e FDA. ${ }^{16}$

A EC ${ }^{12}$ recomenda que no mínimo cinco níveis de concentração (incluindo a amostra zero) sejam empregados na construção da curva analítica. Ainda, estabelece que sejam descritas a faixa de trabalho, a equação matemática e a regressão linear da curva.

De acordo com as recomendações da FDA, ${ }^{16}$ a curva analítica deve ser construída usando uma amostra branco, amostra zero (amostra branco adicionada do padrão interno) e mais matrizes fortificadas com o analito para seis a oito níveis de concentração, abrangendo a faixa de concentração esperada e incluindo o LOQ. Como critérios de avaliação, a FDA recomenda que os resultados devam ser analisados por métodos estatísticos (regressão linear pelo método dos mínimos quadrados) e que deva ser usado o modelo mais simples que adequadamente descreve a relação concentração-resposta. $\mathrm{O}$ uso de outros modelos mais complexos deve ser justificado. Ainda, estabelece que os desvios sejam menores ou iguais a 20 e $15 \%$ em relação à concentração nominal para o LOQ e à concentração nominal para as outras concentrações da curva analítica, respectivamente (no mínimo quatro dos seis níveis de concentração da curva analítica devem atender esses critérios, incluindo o LOQ e a maior concentração da curva analítica).

A ANVISA ${ }^{4}$ segue as recomendações da FDA $^{16}$ apontadas acima e acrescenta que devem ser apresentados os coeficientes linear e angular, o intercepto da reta e que o coeficiente de correlação linear deve ser igual ou superior a 0,98 .

A IUPAC ${ }^{17}$ recomenda que os níveis de concentração da curva analítica devem ser igualmente espaçados entre si, estar sobre a faixa de concentração de interesse, e abranger a faixa de 0 a $150 \%$ do valor esperado. Os padrões analíticos devem ser analisados no mínimo em duplicata, e em ordem aleatória.

Cabe destacar que para avaliar a linearidade do método analítico, os cálculos da regressão linear não são suficientes sendo adequado avaliar também os valores dos resíduos da regressão linear. Se os valores desses resíduos estiverem aleatoriamente distribuídos ao longo da linha da regressão, então a linearidade está confirmada. Uma maneira alternativa de estabelecer a linearidade é plotar um gráfico resposta/concentração versus log da concentração. A linha obtida deve ser horizontal em toda a faixa avaliada, com desvios positivos e negativos para baixas e altas concentrações, respectivamente. Traçando duas linhas paralelas a 95 e $105 \%$ da linha obtida, os pontos de intersecção delimitam a faixa linear do método. ${ }^{18}$

Os procedimentos recomendados pelas agências reguladoras para estabelecer a curva analítica e determinação da linearidade não são harmonizados. Seria desejável especificar que o primeiro nível de concentração da curva analítica seja abaixo do LMR ou 
LMPR estabelecido para o analito.

Outro parâmetro empregado na validação de métodos analíticos e que também é recomendado pela $\mathrm{FAO}^{19}$ é a sensibilidade. A sensibilidade demonstra a variação da resposta em função da concentração do analito e é expressa pelo coeficiente angular da curva analítica. Visto que usualmente a resposta do sinal envolve unidades arbitrárias, a sensibilidade não é considerada um parâmetro de validação nas diretrizes oficiais da EC, ${ }^{12} \mathrm{FDA}^{16}$ e ANVISA. ${ }^{4}$ No entanto, a sensibilidade é representada de forma indireta pela descrição da equação obtida através da regressão linear da curva analítica.

\section{Efeito matriz}

Do ponto de vista da validação de métodos cromatográficos, os requerimentos necessários para a validação estabelecidos pelas agências reguladoras são basicamente os mesmos, independentemente do sistema de detecção utilizado. No entanto, quando a espectrometria de massas for empregada como sistema de detecção em associação com a cromatografia líquida, algumas considerações devem ser feitas em relação ao efeito matriz, uma vez que a matriz pode causar uma supressão da eficiência de ionização e, conseqüientemente, pode ocorrer uma redução na sensibilidade do método. ${ }^{20} \mathrm{~A} \mathrm{FDA}^{16}$ recomenda que esse parâmetro deva ser avaliado para validação de métodos empregando a LC-MS ou LC-MS-MS para garantir que a precisão, seletividade e sensibilidade não sejam afetadas. A ANVISA não faz menção sobre a avaliação do efeito matriz. ${ }^{4}$

Segundo Rogatsky e Stein, ${ }^{20}$ a extensão do efeito matriz na LCMS-MS pode ser avaliada da seguinte forma: adicionar o analito a uma amostra branco e comparar a razão sinal-ruído, área ou altura do pico obtido pelo método com o analito adicionado ao solvente puro; quando não se tiver disponível uma matriz branco pode-se realizar a comparação mediante adição de um padrão interno marcado isotopicamente à amostra e ao solvente puro.

Segundo a recomendação de Rogatsky e Stein, ${ }^{20}$ não fica explícito se a adição do analito deve ser realizada antes do preparo da amostra ou no extrato final a ser injetado no LC-MS. Nesse sentido, é importante ressaltar que para avaliar o efeito matriz, deve ser comparado o sinal obtido para o(s) analito(s) que foi adicionado na matriz após esta já ter passado pelo procedimento de extração, com o sinal do analito no solvente (solução padrão). Se for comparado o sinal obtido da análise da amostra branco que tenha sido fortificada com o(s) analito(s) antes do preparo da mesma, com o sinal do analito no solvente, o que estará sendo avaliado de fato será o efeito matriz mais a eficiência de extração.

\section{Precisão}

Segundo a IUPAC,${ }^{17}$ a precisão corresponde ao grau de concordância de resultados de testes independentes obtidos sob condições estabelecidas e é expressa pela estimativa do desvio padrão (s) ou estimativa do desvio padrão relativo (RSD). Para a validação de métodos em um único laboratório (single-laboratory validation), que é o enfoque proposto para esta revisão, duas etapas são relevantes para esse parâmetro: precisão intra-ensaio: sob condições de repetibilidade, descreve as variações observadas durante uma única corrida analítica (desvio padrão $\sigma_{\text {intra }}$ ) e, precisão inter-ensaios: descreve o grau de variações observadas em diferentes corridas analíticas (desvio padrão $\sigma_{\text {inter }}$ ). As combinações dos resultados obtidos compõem a precisão total do método (Equação 10):

$$
\sigma_{T O T}=\left(\frac{\sigma_{\text {int } r a}^{2}}{n}+\sigma_{\text {inter }}^{2}\right)^{1 / 2}
$$

onde, $\mathrm{n}$ corresponde ao número de repetições para a obtenção de $\sigma_{\text {intra }}$.
Ambas precisões podem ser estimadas mediante análise, em duplicata, da amostra teste, em um número sucessivo de determinações. As componentes individuais das variâncias podem ser calculadas pela análise de variâncias unilateral (ANOVA). ${ }^{17}$ De forma alternativa, a precisão total $\sigma_{\text {Тот }}$ pode ser estimada diretamente através da análise da amostra em determinações sucessivas, mediante avaliação da estimativa do desvio padrão (s).

A IUPAC acrescenta que os resultados obtidos não devem exceder $30 \%$ do valor central.

Assim como a IUPAC, ${ }^{17}$ a FDA ${ }^{16}$ e a ANVISA $^{4}$ também subdividem o parâmetro precisão em intra e inter ensaios. Recomendam para a composição dos ensaios a seleção de pelo menos três diferentes concentrações em torno da concentração de interesse; um mínimo de cinco determinações para cada concentração analisada $(n=5)$. Expressando a precisão em termos de coeficientes de variação $(\mathrm{CV})$, a FDA e a ANVISA recomendam que os resultados não excedam $15 \%$ de CV (exceto para o limite de quantificação, o qual não deve exceder $20 \%$ do CV).

Segundo a EC,,$^{12}$ a precisão é avaliada através de repetibilidade, reprodutibilidade intra-laboratorial e da reprodutibilidade. A seguir são delineados os procedimentos recomendados pela EC para cada um dos parâmetros:

\section{Repetibilidade}

Preparar um conjunto de amostras da mesma matriz em três níveis de concentração, equivalentes a 1; 1,5 e 2 vezes o LMPR ou 0,5; 1 e 1,5 vezes o LMR; promover a análise das amostras fortificadas em cada nível pelo menos com seis replicatas; repetir o procedimento em duas outras ocasiões; calcular a concentração média, a estimativa do desvio padrão e o coeficiente de variação.

\section{Reprodutibilidade intra-laboratorial}

O procedimento e os cálculos são muito semelhantes aos descritos anteriormente para avaliar a repetibilidade, com a diferença de que se pode incluir a fortificação em matrizes diferentes, e que o procedimento deve ser repetido em duas outras ocasiões com diferentes analistas e em diferentes condições, como diferentes reagentes (diferentes procedências), solventes e/ou instrumentos.

\section{Reprodutibilidade}

Parâmetro obtido envolvendo o estudo colaborativo (participação de diferentes laboratórios).

Como critérios de aceitação a $\mathrm{EC}^{12}$ estabelece que o coeficiente de variação $(\mathrm{CV})$, obtido para os ensaios inter-laboratoriais (reprodutibilidade) envolvendo repetidas análises com o material de referência ou amostra branco fortificada, não deve exceder o nível calculado pela Equação de Horwitz (Equação 11):

$C V=2^{(1-0,5 \log C)}$

onde: C é a fração de massa expressa como exponencial de 10 (por exemplo, $1 \mathrm{mg} \mathrm{g}^{-1}=10^{-3}$ ).

$\mathrm{A} \mathrm{EC}^{12}$ salienta ainda que a Equação de Horwitz não deve ser usada para estabelecer um parâmetro de $\mathrm{CV}$ para concentrações inferiores a $100 \mu \mathrm{g} \mathrm{kg}^{-1}$, uma vez que os valores seriam extremamente elevados e que, nesses casos, o CV deve ser tão baixo quanto possível. Já para os ensaios de repetibilidade intra-laboratiorial, o CV deve se situar entre $1 / 2$ e $2 / 3$ do valor calculado pela Equação de Horwitz (11), o que é justificado uma vez que esta equação expressa a dependência entre a variância e a concentração do analito, assumindo resultados obtidos por métodos diferentes em ensaios inter-laboratoriais. Resultados obtidos para a reprodutibilidade intralaboratorial não devem apresentar um $\mathrm{CV}$ superior ao da 
reprodutibilidade inter-laboratorial.

Os termos definidos por algumas agências reguladoras misturam os termos de repetibilidade e reprodutibilidade, o que pode levar a equívocos de conceito. Segundo a IUPAC, ${ }^{17}$ a reprodutibilidade é a concordância entre resultados independentes obtidos com um mesmo método e material teste em diferentes condições (analistas, equipamentos, laboratórios e/ou tempo). No entanto, segundo a FAO, ${ }^{19}$ a maneira mais adequada de avaliar a reprodutibilidade é mediante o ensaio de proficiências (estudos colaborativos entre laboratórios). Para tanto, devem ser utilizados no mínimo cinco materiais testes e a participação de oito laboratórios, recomendação esta que muitas vezes não é passível de ser executada. Não sendo possível o estudo colaborativo pode-se estimar a reprodutibilidade através da precisão inter-ensaio. Guias para ensaios de proficiência podem ser encontradas junto à IUPAC ${ }^{21} \mathrm{e}$ ao INMETRO. ${ }^{22}$

\section{Exatidão}

A exatidão de um método analítico é a proximidade dos resultados obtidos pelo método em estudo em relação ao valor verdadeiro, usando um procedimento experimental para uma mesma amostra por repetidas vezes. ${ }^{17} \mathrm{~A}$ exatidão, quando aplicada a uma série de resultados de ensaio, implica numa combinação de componentes de erros aleatórios e sistemáticos (tendência ou bias). ${ }^{5}$

A exatidão pode ser obtida mediante uso de material de referência certificado (MRC), comparação de métodos ou, ensaios de recuperação. ${ }^{23}$

Para os métodos destinados à análise de alimentos quanto à presença de resíduos de medicamentos veterinários, a exatidão tem sido avaliada costumeiramente mediante o teste de recuperação, visto que existem poucos MRC disponíveis. $\mathrm{O}$ ensaio de recuperação, conforme definições das agências reguladoras IUPAC, ${ }^{17} \mathrm{FAO}^{19}$ e EC, ${ }^{12}$ é realizado mediante adição do analito em diferentes concentrações nas amostras branco. As análises são realizadas conforme procedimento estabelecido e as respectivas concentrações experimentais são calculadas pela curva analítica.

É importante reconhecer que o analito presente na amostra pode ter um comportamento diferente (no preparo de amostra que envolve etapas de extração, separação, purificação e concentração) do que quando o mesmo é adicionado sobre a amostra branco, principalmente quanto se trata de medicamentos veterinários. Esse fato precisa ser levado em consideração na interpretação do resultado obtido no ensaio de recuperação. Ainda, deve-se entender que o valor de recuperação depende do nível de fortificação, no entanto, como a curva analítica também é obtida mediante fortificação de amostras branco, o valor de recuperação acaba refletindo a precisão do método nos diferentes níveis de concentração.

Existe uma grande controvérsia quanto aos termos recuperação e eficiência de extração. As agências reguladoras FDA $^{16}$ e ANVISA $^{4}$ também recomendam o uso da recuperação para obter a exatidão do método, no entanto, definem recuperação como sendo a eficiência de extração do método. Essas agências expressam a recuperação como a percentagem de uma quantidade conhecida do analito alvo, obtida da relação entre os resultados das análises de amostras branco acrescidas de padrão e submetidas ao processo de extração, com os resultados das análises das soluções padrão não extraídas. A ANVISA ${ }^{4}$ recomenda ainda que a percentagem de recuperação seja próxima a $100 \%$, no entanto, admite também valores menores desde que a recuperação seja precisa e exata.

$\mathrm{A} \mathrm{EC}^{12}$ propõe que a exatidão (trueness) seja preferencialmente obtida pelo uso de MRC: proceder à análise de seis replicatas de MRC conforme o método desenvolvido; analisar as amostras e obter as concentrações de cada replicata a partir da curva analítica, cal- cular a média, a estimativa do desvio padrão e o $\mathrm{CV}$ das medidas, e calcular a exatidão pela Equação 13:

$$
\text { Exatidão }(\%)=100\left(\frac{\text { Concentração Média Medida }}{\text { Concentração de MRC }}\right)
$$

No entanto, quando não se tem disponível um MRC, sugere-se o uso do ensaio de recuperação como parâmetro de avaliação da exatidão (recovery). Para tanto, recomenda-se: selecionar 18 alíquotas de uma amostra branco e fortificar em três níveis de concentração em sextuplicata ( $1 ; 1,5$ e 2 vezes o LMPR ou 0,$5 ; 1$ e 1,5 vezes o LMR); analisar as amostras e obter as concentrações de cada replicata a partir da curva analítica, calcular a média, a estimativa do desvio padrão e o CV das medidas, e calcular a recuperação pela Equação 14:

$$
\text { Re cuperação }(\%)=100\left(\frac{\text { Concentração Média Medida }}{\text { Concentração Fortificada }}\right)
$$

A EC ${ }^{12}$ apresenta requerimentos mínimos para os valores de exatidão dos métodos quantitativos de análise de resíduos em alimentos, que variam conforme a concentração de interesse da substância alvo (Tabela 2).

Tabela 2. Exatidão mínima requerida para métodos quantitativos segundo a EC

\begin{tabular}{lc}
\hline Concentração do Analito & Variação na Exatidão \\
\hline$\leq 1 \mu \mathrm{g} \mathrm{kg}^{-1}$ & $-50 \mathrm{a}+20 \%$ \\
$>1 \mu \mathrm{g} \mathrm{kg}^{-1}$ até $10 \mu \mathrm{g} \mathrm{kg}^{-1}$ & $-30 \mathrm{a}+10 \%$ \\
$\geq 10 \mu \mathrm{g} \mathrm{kg}^{-1}$ & $-20 \mathrm{a}+10 \%$ \\
\hline
\end{tabular}

A FDA $^{16}$ e a ANVISA ${ }^{4}$ recomendam que a exatidão seja determinada utilizando-se três níveis de concentração, contemplando a faixa linear da curva analítica, realizando-se no mínimo cinco determinações por concentração. O desvio não deve exceder $15 \%$, exceto para o LOQ para o qual se admite desvios menores ou iguais a $20 \%$. A ANVISA ${ }^{4}$ ainda recomenda que a exatidão seja determinada em uma mesma corrida (exatidão intra-corrida) e em corridas diferentes (exatidão inter-corridas).

Deve-se dar preferência em avaliar a exatidão a partir da análise de um MRC, uma vez que a recuperação avaliada mediante fortificação da amostra pode não reproduzir a mesma situação quando o analito já está presente na amostra.

Frente às controvérsias quanto aos conceitos e forma de avaliar dos parâmetros recuperação e eficiência de extração, ressaltamos que a eficiência de extração é um parâmetro que deve ser avaliado no desenvolvimento do método, no sentido de otimização do mesmo e não deve ser considerado um parâmetro de validação.

\section{Limite de detecção}

Em termos gerais, o limite de detecção é a menor quantidade ou concentração do analito na amostra que pode ser diferenciada, de forma confiável, do zero ${ }^{17}$ ou do ruído de fundo. ${ }^{4} \mathrm{O}$ LOD é definido como sendo a menor quantidade do analito presente em uma amostra que pode ser detectada, porém não necessariamente quantificada, sob as condições experimentais estabelecidas.

Existem controvérsias quanto ao estabelecimento e definição do LOD, assim como, muitas vezes, o LOD do equipamento (detectabilidade) tem sido utilizado de forma incorreta para expressar o LOD do método ou a sensibilidade do mesmo. Segundo o 
INMETRO ${ }^{5}$ o LOD do equipamento é definido como a concentração do analito que produz um sinal de três a cinco vezes a razão ruído/sinal do equipamento, enquanto o LOD do método é definido como a concentração mínima de uma substância, na matriz, medida e declarada com 95 ou $99 \%$ de confiança de que a concentração do analito é maior que zero.

Existem diferentes procedimentos para estimar o LOD, entre esses o método visual, razão sinal-ruído e a partir da curva analítica: ${ }^{13}$ a ANVISA ${ }^{4}$ sugere apenas que o LOD deve ser estabelecido por meio da análise de soluções de concentrações conhecidas e decrescentes do analito, até o menor nível detectável, recomendando que o LOD seja 2 a 3 vezes superior ao ruído da linha de base, no entanto, não fazendo referência se o estudo deve ser realizado com a matriz e o número de replicatas que devem ser realizadas.

A FDA $^{16}$ não menciona o procedimento a ser realizado para estimar o LOD. A IUPAC ${ }^{17}$ recomenda que se estime o LOD a partir de pelo menos seis determinações independentes do analito na amostra branco ou em uma amostra contendo o analito em uma concentração baixa, no entanto, distinguível de zero ou resultado negativo, sendo o valor de LOD estabelecido pelo cálculo de três vezes a estimativa do desvio padrão das medidas.

As recomendações da $\mathrm{EC}^{12}$ não contemplam o LOD nem o limite de quantificação (LOQ) na validação do método destinado para a determinação de resíduos de medicamentos veterinários em alimentos. No entanto, estabelecem a avaliação dos parâmetros de limite de decisão $(\mathrm{CC} \alpha)$ e capacidade de detecção $(\mathrm{CC} \beta)$, que serão discutidos adiante.

\section{Limite de quantificação}

A definição segundo a IUPAC ${ }^{17}$ para o limite de quantificação (LOQ) é a menor quantidade do analito em uma amostra que pode ser determinada com precisão e exatidão aceitáveis sob as condições experimentais estabelecidas. Assim como ocorre para o LOD, existem diversas formas de se estabelecer o LOQ. ${ }^{13}$

Segundo o INMETRO, ${ }^{5}$ o LOQ pode ser considerado como sendo a concentração do analito correspondente ao valor da média do branco mais 5, 6 ou 10 desvios padrão. Algumas vezes é também denominado "limite de determinação", no entanto, a terminologia limite de quantificação é mais usada.

Como na prática o LOQ deve corresponder ao primeiro nível de concentração da curva analítica, algumas agências reguladoras, $\mathrm{ANVISA}^{4}$ e FDA, ${ }^{16}$ têm substituído o termo LOQ por limite inferior de quantificação (LIQ) ou lower limit of quantification (LLOQ), respectivamente.

Para que o primeiro nível de concentração possa ser aceito como LOQ, os seguintes requisitos devem ser atendidos: ${ }^{12}$ a resposta para o analito deve ser de pelo menos cinco vezes a resposta obtida para a amostra branco; a resposta (pico cromatográfico) deve ser identificável e reprodutível com uma precisão de $20 \%$ e exatidão de 80 a $120 \%$.

A ANVISA ${ }^{4}$ segue as recomendações da FDA $^{16}$ e acrescenta que o LOQ também pode ser obtido por meio da análise da amostra branco adicionada de concentrações decrescentes do analito até o menor nível quantificável com precisão e exatidão aceitáveis. Propõe, ainda, para o estabelecimento do LOQ o emprego da razão de 5:1 para o sinal e ruído da linha de base.

É importante ressaltar para que o método seja aceitável para a determinação de resíduo de medicamentos veterinários em alimentos, o LOQ deve ser menor que o LMR ou LMPR estabelecido para o analito (contaminante) em questão.

Limite de decisão (CC $\alpha)$

O limite de decisão $(\mathrm{CC} \alpha)$ é um termo usado pela Comunidade
Européia $^{12}$ e depende de se a substância (medicamento veterinário) a ser analisada na matriz (alimento) possui um LMR ou apenas um LMPR estabelecido.

O limite de decisão é definido como o menor nível de concentração no qual o método pode discriminar com uma certeza estatística de 1- $\alpha$ que o analito em questão está presente. Para as substâncias que apresentam LMR ou LMPR o valor de $\alpha$ é considerado 5 ou $1 \%$, respectivamente.

Para as substâncias que apresentam um LMR, a determinação do $\mathrm{CC} \alpha$ pode ser realizada por dois procedimentos distintos: analisar pelo menos 20 amostras branco por matriz, fortificadas com o analito ao nível do LMR. A concentração no LMR mais 1,64 vezes o desvio padrão correspondente equivale ao $\operatorname{CC} \alpha(\alpha=5 \%)$; fortificar amostras branco em níveis de concentração eqüidistantes ao redor do LMR, analisar as mesmas e plotar uma curva analítica. O valor de CC $\alpha$ será igual ao LMR (concentração) mais 1,64 vezes o desvio padrão da reprodutibilidade intra-laboratorial $(\alpha=5 \%)$.

Para as substâncias que possuem um LMPR, o CC $\alpha$ pode ser estabelecido por dois procedimentos distintos: analisar pelo menos 20 amostras branco por matriz e calcular a razão sinal-ruído na faixa do tempo de retenção do analito. O $\mathrm{CC} \alpha$ é três vezes a razão sinal ruído; fortificar amostras branco ao nível do LMPR e acima deste valor em concentrações eqüidistantes. Analisar as amostras e plotar um gráfico do sinal em função da concentração adicionada. A concentração correspondente ao intercepto e mais 2,33 vezes o desvio padrão da reprodutibilidade intra-laboratorial do intercepto equivale ao $\operatorname{CC} \alpha(\alpha=1 \%)$.

\section{Capacidade de detecção $(C C \beta)$}

A capacidade de detecção representa a menor quantidade da substância que pode ser detectada, identificada e/ou quantificada em uma amostra com uma probabilidade de erro aceitável $(\beta) .^{12}$

Como para $\mathrm{CC} \alpha$, o estabelecimento da $\mathrm{CC} \beta$ depende também se a substância apresenta um valor de LMR estabelecido ou apenas possui um LMPR.

Para as substâncias que apresentam um LMR, a determinação do CC $\beta$ pode ser realizada por dois procedimentos distintos: analisar pelo menos 20 amostras branco por matriz, fortificadas com o analito no limite de decisão $(\mathrm{CC} \alpha)$. O valor de $\mathrm{CC} \alpha$ mais 1,64 vezes o desvio padrão correspondente equivale ao $\operatorname{CC} \beta(\beta=5 \%)$; fortificar amostras branco em níveis de concentração eqüidistantes ao redor do LMR, analisar as mesmas e plotar uma curva analítica. Calcular o desvio padrão na concentração correspondente ao $\mathrm{CC} \alpha$. O valor de $\operatorname{CC} \beta$ será igual ao valor de $\mathrm{CC} \alpha$ (concentração) mais 1,64 vezes o desvio padrão da reprodutibilidade intra-laboratorial $(\beta=5 \%)$.

Sustâncias para as quais há LMPR estabelecido, o CC $\beta$ pode ser determinado das seguintes formas: analisar pelo menos 20 amostras branco por matriz, fortificadas com o analito na concentração do $\mathrm{CC} \alpha$. O $\mathrm{CC} \beta$ é igual ao $\mathrm{CC} \alpha$ mais 1,64 vezes o desvio padrão da reprodutibilidade intra-laboratorial $(\beta=5 \%)$; fortificar amostras branco em níveis de concentração eqüidistantes ao redor do LMPR. Analisar as amostras e plotar um gráfico do sinal em função da concentração adicionada. A concentração correspondente ao $\mathrm{CC} \alpha$ mais 1,64 vezes o desvio padrão da reprodutibilidade intra-laboratorial da média mensurada contendo $\operatorname{CC} \alpha$ equivale ao $\operatorname{CC} \beta(\beta=5 \%)$.

Vale observar que para as substâncias que possuem LMPR estabelecido, o $\mathrm{CC} \alpha$ e o $\mathrm{CC} \beta$ correspondem ao LOD e LOQ, respectivamente. Para as substâncias com LMR estabelecido não existe relação entre o $\mathrm{CC} \alpha$ e o $\mathrm{CC} \beta$ e os LOD e LOQ, mas fazem referência ao valor de LMR e são importantes para inspeção de produtos de origem animal quanto à presença de resíduos de medicamentos veterinários. 


\section{Robustez}

A robustez de um método de ensaio mede a sensibilidade que este apresenta face a pequenas variações. ${ }^{5} \mathrm{Um}$ método diz-se robusto quando se revelar praticamente insensível a pequenas variações que possam ocorrer quando o mesmo está sendo executado. As agências reguladoras empregam diferentes termos para expressar a robustez do método: ruggedness ${ }^{12,14,17}$ e robustness ${ }^{13}$. Cabe ressaltar que embora esses dois termos tenham sido empregados, não existe uma diferença clara entre os mesmos, o que pode levar a uma confusão na interpretação das definições, assim como não está estabelecido se o parâmetro robustez deve ser avaliado durante o

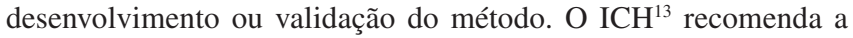
avaliação da robustez (robustness) no desenvolvimento do método e sugere como variáveis a serem avaliadas para um método de cromatografia líquida de alta eficiência: pH da fase móvel, composição da fase móvel, colunas de diferentes fornecedores ou lotes, temperatura e vazão.

Enquanto a FDA $^{16}$ e a ANVISA $^{4}$ não incluem a robustez na validação de métodos destinados à análise de resíduos de medicamentos veterinários em alimentos, a $\mathrm{EC}^{12}$ recomenda a avaliação deste parâmetro (ruggedness), empregando um planejamento fatorial (teste de Youden). Neste teste são realizadas oito combinações de sete fatores de variáveis. No entanto, a $\mathrm{EC}^{12}$ cita como exemplos de variáveis diferentes espécies, diferentes matrizes e condições de amostragem, que são consideradas fontes de maiores variações nos resultados e, embora sejam relevantes, não se enquadram na definição do termo robustez.

\section{CONCLUSÕES}

Indubitavelmente a validação é imprescindível para garantir a confiabilidade dos resultados de um determinado procedimento analítico. Considerando o objetivo da determinação de medicamentos veterinários em alimentos, cada país segue as normas estabelecidas pela agência reguladora oficial, no caso do Brasil, a ANVISA do Ministério da Saúde. Os procedimentos recomendados pela ANVISA para validação de métodos bioanalíticos seguem quase que integralmente as recomendações adotadas pela agência dos Estados Unidos, a FDA. Contudo, cabe salientar que as recomendações, de modo geral, entre os Estados Unidos da América, a Comunidade Européia e o Brasil não são padronizadas. A Comunidade Européia faz uso de um protocolo de validação diferenciado em relação à FDA e, embora os parâmetros sejam praticamente os mesmos, os procedimentos para obtenção dos mesmos se diferenciam o que dificulta a sua interpretação. Ainda, alguns termos como estabilidade do analito na matriz e eficiência de extração são considerados por algumas agências reguladoras como parâmetros a serem avaliados no desenvolvimento do método, enquanto para outras são consideradas como parte integrante do processo de validação. Do mesmo modo, existem controvérsias na obtenção e interpretação dos termos ensaios de recuperação para avaliação da exatidão e eficiência de extração. É importante que esses conceitos sejam revistos e harmonizados.

Agências reguladoras importantes a serem consideradas na validação de métodos, embora não sejam destinados especificamente para análise de resíduos de medicamentos veterinários em alimentos, são o INMETRO e a IUPAC. Ambas entidades apresen- tam terminologias, definições e procedimentos rigorosamente detalhados e coerentes.

Um aspecto importante a ser considerado em se tratando de métodos destinados à quantificação de resíduos de medicamentos veterinários em alimentos é o fato de que apenas garantir a confiabilidade analítica das medidas não é suficiente. Para tanto, na validação deve ser levado em consideração o LMR ou LMPR da substância alvo, no intuito de garantir que o método seja adequado para o objetivo proposto, ou seja, no controle da qualidade de alimentos visando a inocuidade alimentar. Enquanto a Comunidade Européia cumpre essa exigência, os protocolos adotados pela FDA e ANVISA não deixam evidente a importância desses limites no estabelecimento dos parâmetros de validação.

De modo geral, os protocolos de validação não levam em consideração a técnica analítica envolvida, embora a maioria cite o uso de métodos cromatográficos, sem, no entanto, considerar as diferenças e peculiaridades do sistema de detecção envolvido. Considerando que para a determinação de resíduos de medicamentos veterinários em alimentos os métodos analíticos mais apropriados são os cromatográficos associados à espectrometria de massas é importante incluir no protocolo o parâmetro efeito matriz.

\section{REFERÊNCIAS}

1. http://www.who.int/foodsafety/en/, acessada em Março 2007.

2. http://www. anvisa.gov.br, acessada em Janeiro 2007.

3. http://www. inmetro.gov.br, acessada em Janeiro 2007.

4. Agência Nacional de Vigilância Sanitária (ANVISA); Guia para Validação de Métodos Analíticos e Bioanalíticos, RE n 899, de 29/05/2003.

5. Instituto Nacional de Metrologia, Normalização e Qualidade Industrial (INMETRO); Orientações sobre Validação de Métodos de Ensaios Químicos, DOQ-CGCRE-008, 2003.

6. http://goldbook.iupac.org/R05305.html, acessada em Janeiro 2007.

7. International Standard Organization; General Requirements for the Competence of Testing and Calibration Laboratories, ISO/EC 17025, 1999.

8. http://www.ich.org/, acessada em Janeiro 2007.

9. International Standard Organization (ISO); Guide to the Expression of Uncertainty in Measurements, Geneva, 1993.

10. EURACHEM; Quantifying Uncertainty in Analytical Measurement, London, 1995.

11. http://jecfa.ilsi.org/section1.htm\#1, acessada em Abril 2007.

12. European Commission; Official Journal of the European Communities, 17/ 08/2002, L221/8-36.

13. International Conference on Harmonization (ICH); Validation of Analytical Procedures: Methodology, Q2B, Step 4, Consensus Guideline, 6 November 1996.

14. United States Pharmacopeia (USP) Convention; US Pharmacopeia 27, Rockville, 2004.

15. Collins, C. H.; Braga, G. L.; Bonato, P. S.; Fundamentos de Cromatografia, Editora UNICAMP: Campinas, 2005.

16. United States Food and Drug Administration (US-FDA), Center for Drug Evaluation and Research, Center for Veterinary Medicine, Department of Health and Human Services; Guidance for Industry, Bioananalytical Method Validation, May, 2001.

17. Thompson, M.; Stephen, L. R.; Wood, R.; Pure Appl. Chem. 2002, 74, 835.

18. Food and Agriculture Organization of the United Nations (FAO); Validation of Analytical Methods for Food Control December, 1997.

19. Huber, L.; LC-GC Int. 1998, 11, 96.

20. Rogatsky, E.; Stein, S.; J. Am. Soc. Mass Spectrom. 2005, 16,1757.

21. Thompson, M.; Ellison, S.R.; Wood, R.; Pure Appl. Chem. 2006, 78, 145.

22. http://www.inmetro.gov.br/kits/ctle05_protocolo.pdf, acessada em Março 2007.

23. Miller J. C.; Miller J. N.; Statistics for Analytical Chemistry, Ellis Horwood; New York, 1993. 